\title{
The Curious Case of a Missing Gallbladder: An Unusual Presentation of a Cholecystoduodenal Fistula
}

\author{
Sarvani Surapaneni ${ }^{1}$ Wissam Kiwan ${ }^{2}$ Michael K. Chiu ${ }^{3} \quad$ Alkis Zingas $^{3}$ Shakir Hussein ${ }^{4}$ S $^{\circ}$ \\ Murray Ehrinpreis ${ }^{5}$ \\ ${ }^{1}$ Internal Medicine Department, Wayne State University, Detroit, \\ Michigan, United States \\ ${ }^{2}$ Gastroenterology Division, University of Southern California, Los \\ Angeles, California, United States \\ ${ }^{3}$ Department of Radiology, Wayne State University, Detroit, \\ Michigan, United States \\ ${ }^{4}$ Department of Surgery, Wayne State University, Detroit, Michigan, \\ United States \\ ${ }^{5}$ Gastroenterology Division, Wayne State University, Detroit, \\ Michigan, United States \\ Address for correspondence Wissam Kiwan, MD, Gastroenterology \\ Division, University of Southern California, 2011 ZONAL AVE, HMR \\ 101, Los Angeles, CA 90089-1015, United States \\ (e-mail: wissam.kiwan@med.usc.edu). \\ Avicenna J Med 2022;12:34-37.
}

Abstract
Keywords
- Cholecystoduodenal
Fistula
- Large Gallstones
- Endoscopy

Large gallstones could erode through gallbladder wall to nearby structures, causing fistulas, gastric outlet obstruction and gallstone ileus. They typically occur in elderly patients with comorbidities carrying therapeutic challenges. We present a case of a middle-aged woman who was thought to have symptomatic cholelithiasis. Extensive adhesions precluded safe cholecystectomy. While hepatobiliary iminodiacetic acid scan and magnetic resonance imaging with cholangiopancreatography (MRI-MRCP) failed to visualize the gallbladder, computed tomography $(\mathrm{CT})$ was consistent with cholecystoduodenal fistula. A very large gallstone was seen endoscopically in the duodenum, which was broken down into pieces using a large stiff snare.

\section{Introduction}

Biliary-enteric fistula is a rare complication of cholelithiasis. It may result from large gallstones eroding through the gallbladder wall to the adjacent intestines or stomach. ${ }^{1,2}$ We present a unique case of a middle-aged woman with a $5-\mathrm{cm}$ gallstone found in the duodenum that caused acute abdominal pain without gastric outlet obstruction (GOO) and was treated endoscopically.

\section{Case Presentation}

A 56-year-old woman with diabetes, hypertension, and morbid obesity presented with acute right upper quadrant and

published online

December 2, 2021
DOI https://doi.org/ 10.1055/s-0041-1739541. ISSN 2231-0770. epigastric pain for three days. It was burning, constant, and associated with nausea but no emesis. There were no aggravating or alleviating factors. The patient had no prior abdominal surgeries. On physical examination, vital signs were normal. Body mass index (BMI) was $48 \mathrm{~kg} / \mathrm{m}^{2}$. Tenderness without rigidity was noted in the right upper guardant, but Murphy's sign was negative. The complete blood count with differential, liver chemistry, serum creatinine, blood urea nitrogen, and lipase were all within normal limits. Ultrasonography showed gallstones in a contracted gallbladder with no signs of cholecystitis, and a normal-size common bile duct. Symptomatic cholelithiasis was diagnosed. Cholecystectomy was recommended; however, the patient chose to decline the surgery at that time and was discharged home.

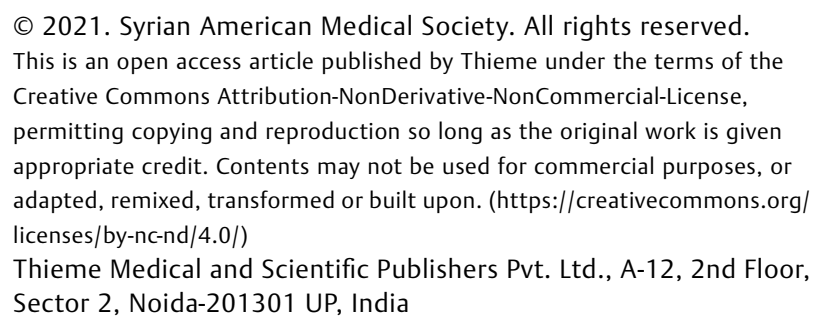


One week later, the patient returned with worsening abdominal pain without change of the vital signs, examination or laboratories. Robotic-assisted laparoscopic cholecystectomy was attempted; however, there was limited access to the gallbladder due to extensive amount of adhesions. Robotic lysis of the omental adhesions to the gallbladder fossa was performed, following which the duodenum and the stomach were found adhered to the gallbladder fossa. Thus, cholecystectomy was deemed unsafe and the procedure was aborted. After the operation, alanine transaminase increased to $226 \mathrm{U} / \mathrm{L}$ (from $9 \mathrm{U} / \mathrm{L}$ ), aspartate transaminase to $228 \mathrm{U} / \mathrm{L}$ (from $10 \mathrm{U} / \mathrm{L}$ ) and alkaline phosphatase to $80 \mathrm{U} / \mathrm{L}$ (from 29 $\mathrm{U} / \mathrm{L}$ ). Total bilirubin increased from 0.35 to $1.90 \mathrm{mg} / \mathrm{dL}$ (direct bilirubin $1.08 \mathrm{mg} / \mathrm{dL}$ ).

A subsequent magnetic resonance imaging cholangiopancreatography (MRI-MRCP) showed minimal fluid in the gallbladder fossa but no evidence of biliary ductal dilation or choledocholithiasis. The gallbladder could not be identified (-Fig. 1). Subsequently, hepatobiliary iminodiacetic acid (HIDA) scan was done but did not visualize the gallbladder either. A computed tomography (CT) scan of abdomen with intravenous (IV) contrast confirmed a cholecystoduodenal fistula along with pneumobilia of a nondilated biliary tree ( - Fig. 2).

On postop day seven, esophagogastroduodenoscopy (190HD, Olympus) revealed a $5-\mathrm{cm}$ brown, smooth, oval and hard stone along with a 1 to $2 \mathrm{~cm}$ stone in the duodenal bulb (-Fig. 3). An inflamed and scarred fistulous opening was seen in the bulb (-Fig. 4). Further advancement of the scope beyond the stones was unsuccessful. The smaller stone was removed using a $3 \times 6 \mathrm{~cm}$ roth net (STERIS, OH). Follow-

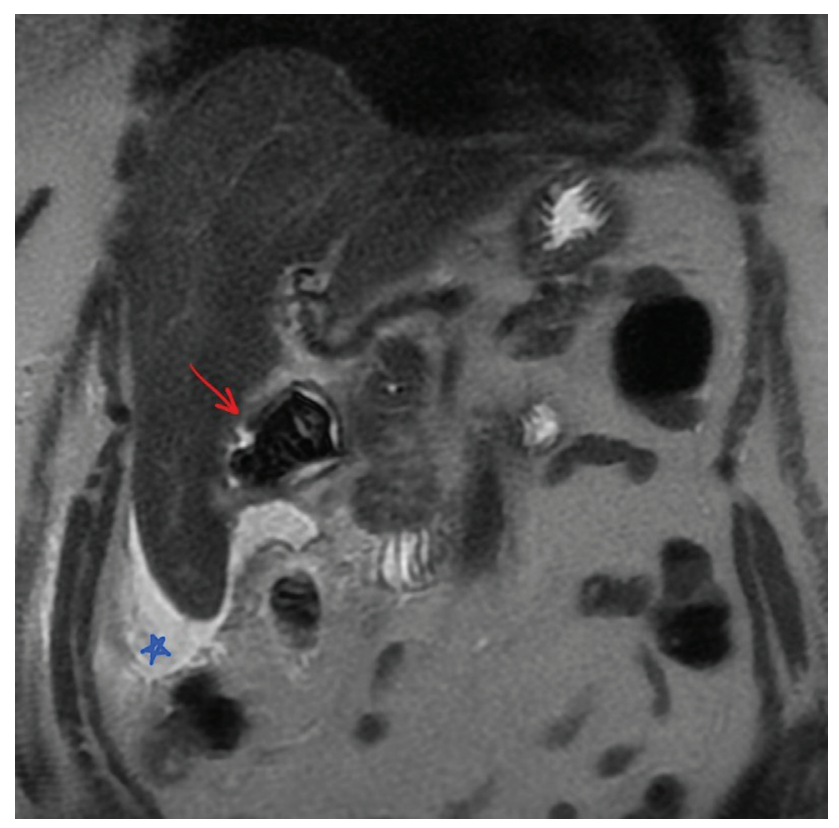

Fig. 1 Coronal T2 single-shot fast spin echo (SSFSE)-weighted magnetic resonance imaging (MRI) of the abdomen demonstrating small amount of hyperintense ascites surrounding the liver (blue star), and a large heterogeneous, predominantly $\mathrm{T} 2$ hypointense signal likely within the duodenal bulb which represents a very large gallstone (red arrow; identified on imaging retrospectively only after endoscopy).

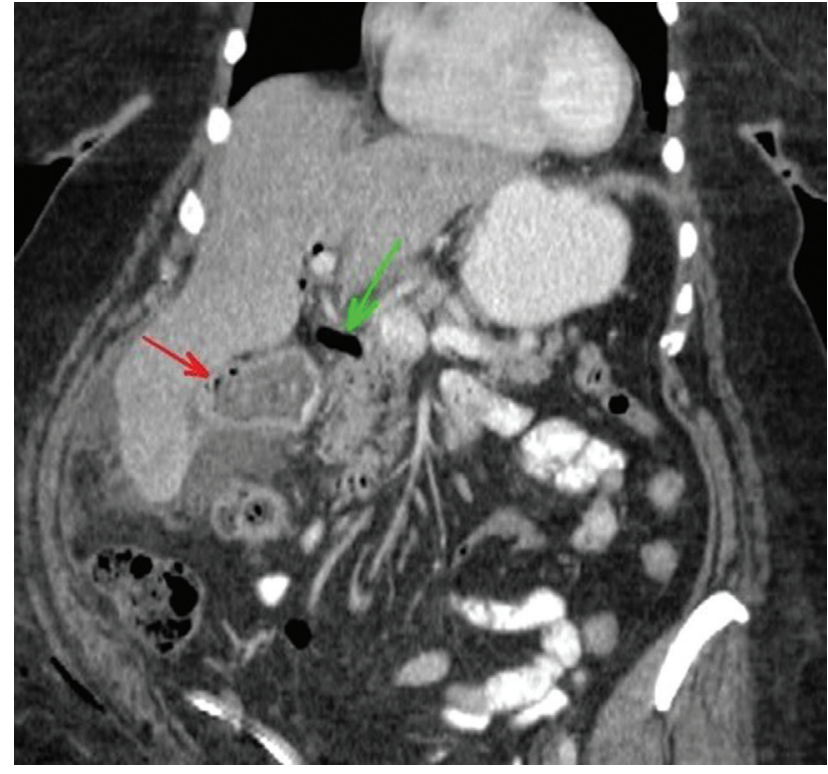

Fig. 2 Coronal, orally-and intravenously enhanced computed tomography (CT) of the abdomen demonstrates a large calculus (red arrow) with a few foci of air as well as air within the biliary system (green arrow), suggesting cholecystoenteric fistula. A small amount of ascites surrounds the liver. Subcutaneous air within the right abdominal wall and adjacent to the gluteal muscles due to recent laparoscopy is noted.

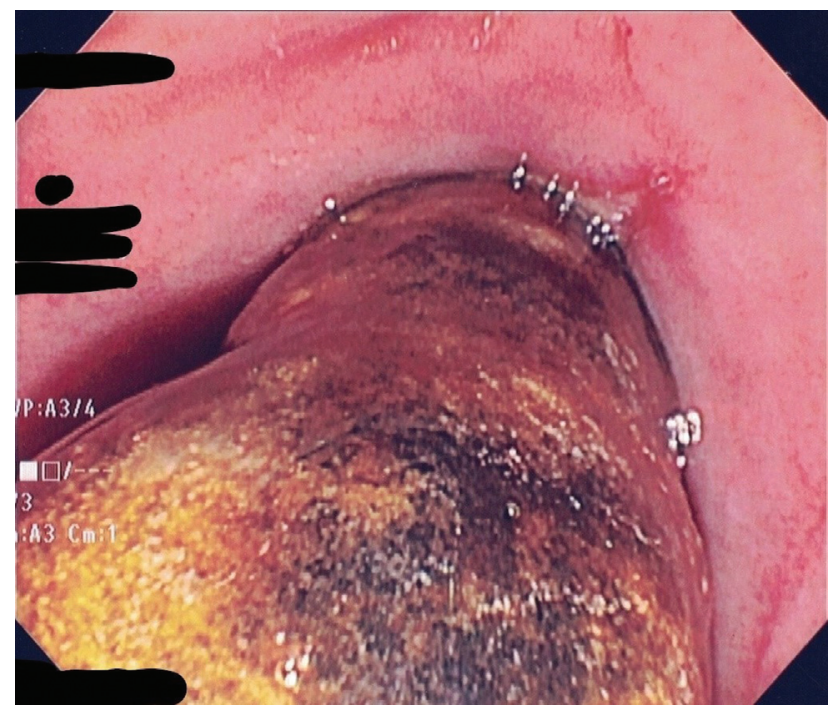

Fig. 3 A very large gallstone was found in the bulb.

ing multiple attempts with roth nets, 3-cm trapezoid biliary stone basket (BostonScientific, MA), less than half of the large stone was fragmented and removed. The larger stone fragment, however, could not be retrieved through the pylorus due to its size. Due to the length of procedure and considering surgical options, the endoscopy was concluded.

To prevent gallstone-induced gastrointestinal obstruction at the ileocecal valve from the leftover large stone fragment, and to avoid an invasive laparotomy in a patient already found to have extensive adhesions due to the fistulization, a multidisciplinary decision was made to attempt endoscopic retrieval again. Due to the lack of specialized lithotripsy 


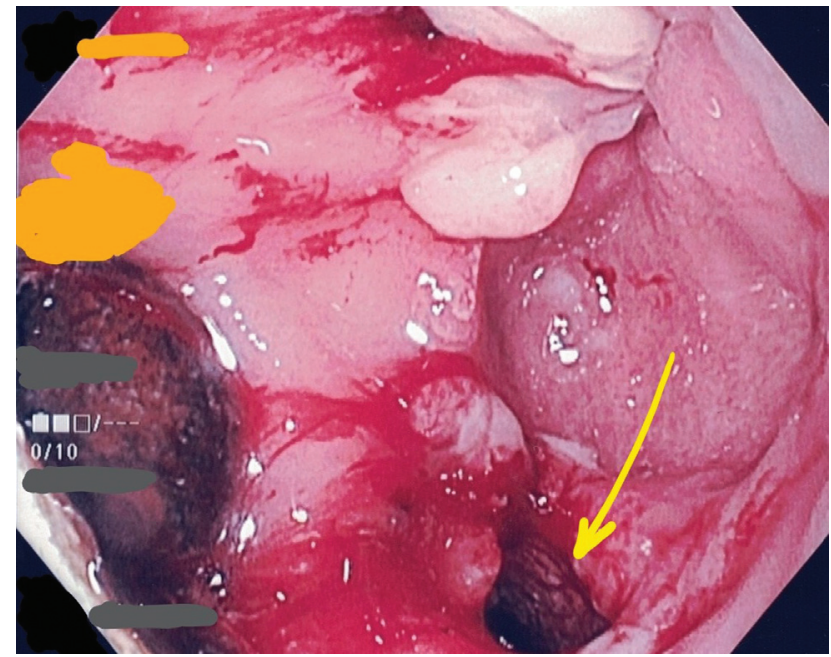

Fig. 4 Fistulous tract opening in the bulb (yellow arrow). Note the epithelization of the tract as well as a stone in the tract or the gallbladder.

equipment, a large snare with a stiff wire (Captivator $27 \mathrm{~mm}$; BostonSicentific, MA) was used to break it down by first confirming that only stone was grasped with the snare (-Fig. 5), then forcefully and quickly pulling it against the plastic tip of the snare. After that, the fragments were brought to stomach for further fragmentation using the same method. This allowed passage of the smaller pieces through esophagus. Caution was exercised to avoid mucosal damage of the sharp edges of the fragments, especially in the esophagus. A total of 15 fragments were retrieved using a roth net, leaving no fragments behind ( - Fig. 6 ). Overtube was considered to protect the esophageal mucosa from the sharp edges of the stones. However, this was deferred after successful fragmentation of the stone to small pieces.

Following the procedure, symptoms improved, bilirubin and liver enzymes normalized, and the patient opted for cholecystectomy in the future if symptoms were to recur. Ciprofloxacin and metronidazole for a total of 2 weeks were

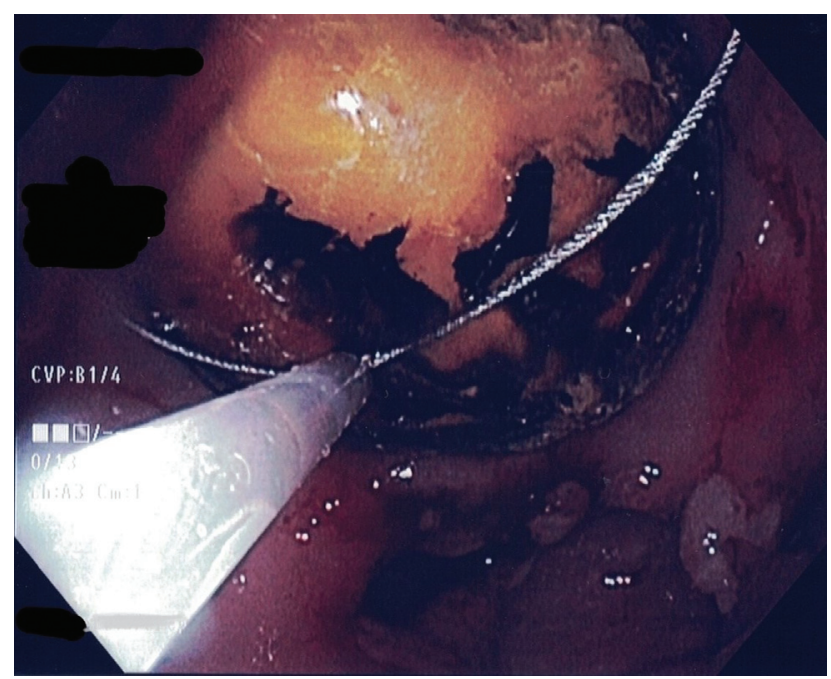

Fig. 5 Fragmentation of the large stone using a stiff snare.

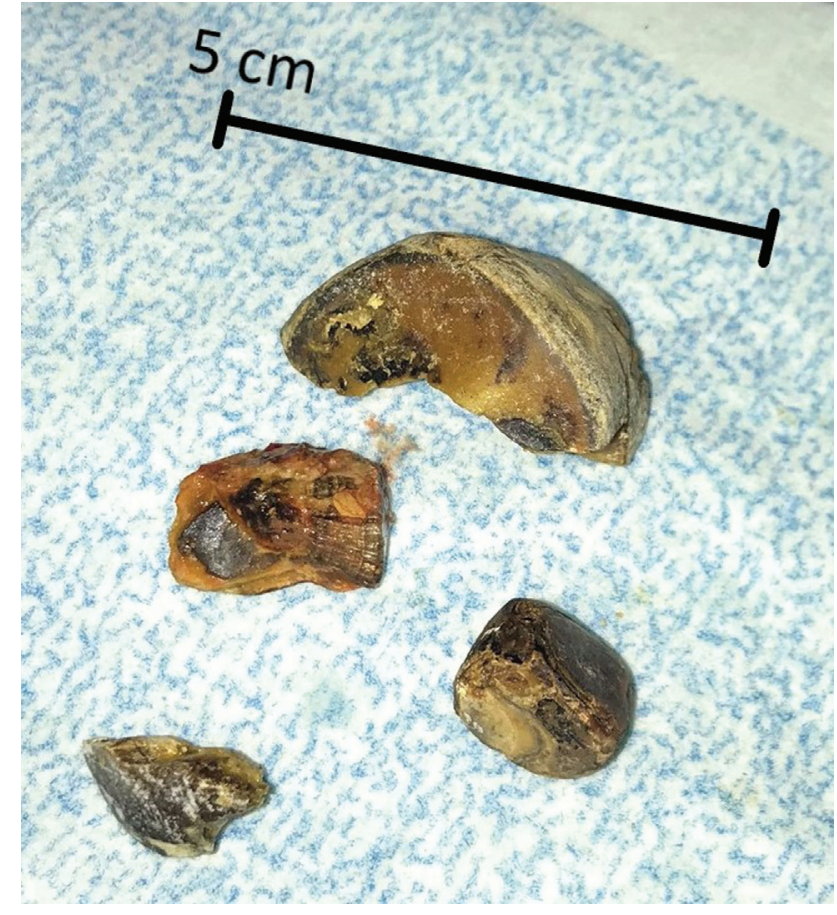

Fig. 6 Some fragments of the large stone after retrieval. Largest fragment is approximately $3 \mathrm{~cm}$ long.

prescribed, and the patient was discharged 3 days after procedure. Eighteen days later, the patient was seen as an outpatient and continued to recover well.

\section{Discussion}

What was initially thought to be a classic case of symptomatic cholelithiasis was found to be a clinically, surgically, and endoscopically challenging cholecystoduodenal fistula. The presentation of this fistula with a very large gallstone at a young age is very unusual. In addition, despite the size of the gallstone (measured at $5 \mathrm{~cm}$ on MRCP retrospectively), the patient had no GOO. The fistula was not identified during an attempted cholecystectomy nor was it seen on the MRCP. Although it is unusual, the substantial burden of adhesions precluded the ability of diagnosing the fistula during the attempted operation. Only CT then endoscopy revealed it. Removal of such a large stone was deemed important due to the ongoing severe abdominal pain, and to prevent impending $\mathrm{GOO}$ and gallstone ileus. A multidisciplinary discussion led to the decision of attempting another endoscopic therapy, to avoid a high-risk surgery in a high-risk patient (extensive intraabdominal adhesions, morbid obesity and other comorbidities).

Biliary-enteric fistulas occur in 2 to $3 \%$ of all cases of cholelithiasis. ${ }^{3}$ They develop due to local ischemia in the wall of the gallbladder with gradual perforation and tract formation. ${ }^{1,2}$ Larger gallstones that could lead to gallstone ileus typically present at older ages with mean age 70 to 75 years old, affecting women more than men. ${ }^{1,2,4}$ In addition to gallstone ileus, impaction of a large stone in the pylorus or duodenum may result in GOO; the hallmark of a rare entity called Bouveret's syndrome., ${ }^{2,5}$ 
Various minimally invasive treatment modalities have been utilized to treat large gallstones. These modalities are more frequently used to treat stones in the bile ducts. Mechanical lithotriptors (out-of-scope and through-the-scope) are devices that require limited training. As the name implies, extracorporeal shock-wave lithotripsy (ESWL) involves concentration of focused shock waves to gradually disintegrate the stones. This method requires radiographic localization of the stones. In electrohydraulic lithotripsy (EHL) and laser lithotripsy (LL), concentrated energy is applied to the stone under direct endoscopic visualization. The decision to choose among these modalities is often made based on stone characteristics (shape, position, and hardness), availability of the modality and local expertise. $^{6}$

While endoscopic treatment could be successful, ${ }^{7}$ often times it is not ${ }^{8-10}$ and surgery becomes inevitable. In a systematic review published in 2017 assessing existing literature on management of Bouveret's syndrome with large gallstones, ${ }^{11}$ endoscopic treatment was attempted in most cases (77\%; 160 cases) with a relatively low success rate of $29 \%$. An endoscopic approach is not devoid of risk of complications such as perforation, stone impaction in the esophagus and gastrointestinal hemorrhage. Notwithstanding, it is reasonable to attempt the minimally invasive upper endoscopic therapeutic interventions prior to surgery, unless there is gallstone ileus. ${ }^{1,2,5,11-15}$

In summary, this was an unusual case of cholecystoduodenal fistula with a large gallstone in the bulb without causing GOO at a relatively young age, which was treated successfully by using readily available stiff wire snares to achieve safe and controlled mechanical lithotripsy.

Note

This case study was presented as a poster at the 2020 Annual American College of Gastroenterology Meeting [virtual], 10/2020.

\section{Authors' Contributions}

The manuscript has been read and approved by all the authors, the requirements for authorship have been met, and each author believes that the manuscript represents honest work. All authors provided substantial contributions to the concept, drafting, and final approval. This manuscript is a result of collaboration among GI, general surgery and radiology.

Funding

None.

Conflict of Interest

None declared.

\section{References}

1 Ayantunde AA, Agrawal A. Gallstone ileus: diagnosis and management. World J Surg 2007;31(06):1292-1297

2 Warren DJ, Peck RJ, Majeed AW. Bouveret's syndrome: a case report. J Radiol Case Rep 2008;2(04):14-17

3 van Hillo M, van der Vliet JA, Wiggers T, Obertop H, Terpstra OT, Greep JM. Gallstone obstruction of the intestine: an analysis of ten patients and a review of the literature. Surgery 1987;101(03):273-276

4 Halabi WJ, Kang CY, Ketana N, et al. Surgery for gallstone ileus: a nationwide comparison of trends and outcomes. Ann Surg 2014; 259(02):329-335

5 Haddad FG, Mansour W, Deeb L. Bouveret's syndrome: literature review. Cureus 2018;10(03):e2299

6 Doshi B, Yasuda I, Ryozawa S, Lee GH. Current endoscopic strategies for managing large bile duct stones. Dig Endosc 2018;30 (Suppl 1):59-66

7 Le Mouel JP, Thiebault H, Hakim S. Bouveret's syndrome. Clin Gastroenterol Hepatol 2019;17(06):e55

8 Ramos GP, Chiang NE. Bouveret's syndrome. N Engl J Med 2018; 378(14):1335

9 Philipose J, Khan HM, Ahmed M, Idiculla PS, Andrawes S. Bouveret's syndrome. Cureus 2019;11(04):e4414

10 Osman K, Maselli D, Kendi AT, Larson M. Bouveret's syndrome and cholecystogastric fistula: a case-report and review of the literature. Clin J Gastroenterol 2020;13(04):527-531

11 Al-Habbal Y, Ng M, Bird D, McQuillan T, Al-Khaffaf H. Uncommon presentation of a common disease - Bouveret's syndrome: A case report and systematic literature review. World J Gastrointest Surg 2017;9(01):25-36

12 Clavien PA, Richon J, Burgan S, Rohner A. Gallstone ileus. Br J Surg 1990;77(07):737-742

13 Doycheva I, Limaye A, Suman A, Forsmark CE, Sultan S. Bouveret's syndrome: case report and review of the literature. Gastroenterol Res Pract 2009;2009:914951

14 Mavroeidis VK, Matthioudakis DI, Economou NK, Karanikas ID. Bouveret syndrome-the rarest variant of gallstone ileus: a case report and literature review. Case Rep Surg 2013;2013:839370

15 Park SH, Lee SW, Song TJ. Another new variant of Bouveret's syndrome. World J Gastroenterol 2009;15(03):378-379 